\title{
The Liquid Crystal State Poliamidbenzimidazola Solutions in Sulfuric Acid
}

\author{
Oleg Khanchich ${ }^{1, *}$ and Svetlana A. Kuznetsova ${ }^{2}$ \\ ${ }^{1}$ Moscow Technological Institute, 119334 Moscow, Russia \\ ${ }^{2}$ Institute of Chemistry and Chemical Technology Russian Academy of Sciences, 660036 Krasnoyarsk, Russia
}

\begin{abstract}
We studied the temperature and concentration conditions of education and the field of LC phase of existence in sulfuric acid solutions poliamidbenzimidazola. The polarization-optical methods and the structural features of biphasic and anisotropic areas and built plots the phase diagram of the concentrated solutions poliamidbenzimidazola in $\mathrm{H} 2 \mathrm{SO} 4$. It is shown that in certain temperature - concentration of cooling modes can be observed the coexistence of three phases: isotropic crystal and a liquid crystal, which is shown as a characteristic of liquid crystal birefringent domains.
\end{abstract}

\section{Introduction}

Currently, a wide importance in mechanical engineering, especially aircraft, purchased polymer composites. They are obtained on the basis of reinforcing fibers and polymer matrices [1]. Used as a template, along with other thermoplastics polyheteroarylenes. The best studied of these is poly-n-phenylene terephthalamide (PPTA) [2]. Fibers of this polymer is formed from a liquid crystal (LC) solutions in concentrated sulfuric acid. Less studied, but no less practical significance is poliamidbenzimidazol (PABI), which is also derived from the $\mathrm{LC}$ in $\mathrm{H}_{2} \mathrm{SO}_{4}$ solutions [3]. Research polyheteroarylenes LC status is of great scientific and practical importance, as it allows to establish the general laws of phase transitions in polymers and to use the transition phenomenon in the mesophase to obtain highstrength fibers and films. The aim of this work was to study the temperature and concentration conditions for transition $\mathrm{PABI} \mathrm{H}_{2} \mathrm{SO}_{4}$ solutions.

\section{Experimental}

In order to measure the temperatures of the liquid crystal $\rightarrow \leftarrow$ isotropic liquid solutions were placed in a sealed cell and asked by a teflon gasket layer of uniform thickness of $0,2 \mathrm{~mm}$ between the glass of the solution. The cuvette was then heated to the complete disappearance of the optical anisotropy and held under these conditions for 10-30 minutes. Control of the process was carried out to achieve a minimum amount of light transmission in crossed polaroid and visually observation of dark field microscope in crossed polaroid (picture blanking) and the complete disappearance of $\mathrm{H}_{v^{-}}$ scattering patterns. This temperature is taken for the transition to an isotropic (I) phase. The difference thus determined temperature values through a variety of polarization-optical methods was no more than $1-2^{\circ}$. The beginning of the reverse transition I $\rightarrow$ LC and the temperature is lowered is the appearance of the light transmission in crossed polaroid. We investigated the solution cooled to $20^{\circ} \mathrm{C}$ and aged after cooling for a time sufficient to complete stabilization of light transmission. It is possible to eliminate the distortions introduced into the polarization-optical studies of stress anisotropy in a high concentrated solution and determine the critical concentration of the transition in the liquid crystal state. To eliminate the influence of crystal solvates the optical properties of the system and the study of phase transformations associated with the formation of LC phases namely, using the fact that the occurrence of crystal solvates due to the large induction period (from several hours to $2-3$ days).

\section{Results and discussion}

The structural formula of this polymer is as follows:

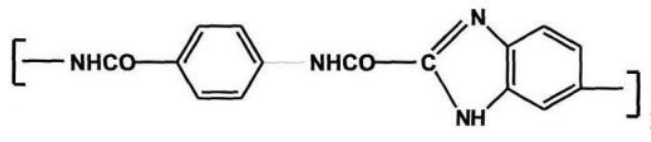

It was found that at concentrations in excess of $11 \%$ solution in $\mathrm{H}_{2} \mathrm{SO}_{4} \mathrm{PABI}$ transformed into anisotropic state, similarly to forming solutions of PPTA typical lyotropic liquid crystal. PABI anisotropic solutions in $98 \% \mathrm{H}_{2} \mathrm{SO}_{4}$ reprecipitated polymer was prepared from an intrinsic viscosity $[\eta]=5,8 \mathrm{dl} / \mathrm{g}$ measured in dimethylacetamide at $20^{\circ} \mathrm{C}$. The solutions have a high viscosity (over $10^{6}$ poise) were opaque and exhibited deformation characteristic "whitish", which disappeared after a while. Externally, the sulfuric acid solution with the concentration changed color from brown to whitish. In a sealed cell solutions show an interference pattern

Corresponding author: o hanchich@mti.edu.ru 
suppression, which disappears on heating and reappears on cooling.

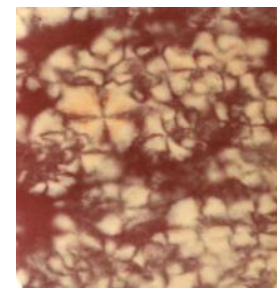

$12 \%$

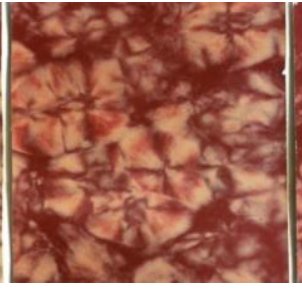

$14 \%$

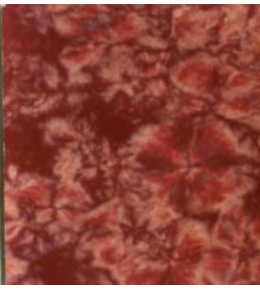

$16 \%$
Fig. 1. The micrographs in crossed nicols of LC solutions of different concentrations PABI

Light scattering from such a sample has the form of a centrally symmetric spots, similar to the solution of the PPTA unoriented LC, with the intensity of the same order in $H_{v}$ and $V_{v}$ polarizations (Fig. 2, $H_{v}$, and $V_{v}$, a) that indicates the predominance of orientation fluctuations in the system and is typical of liquid crystal.

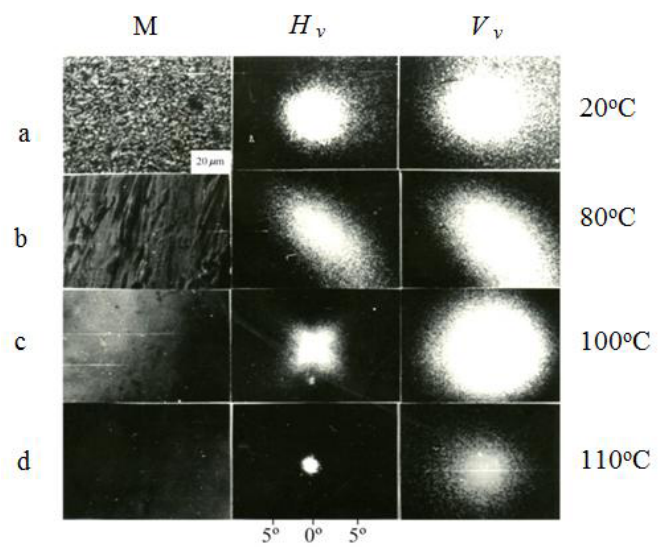

Fig. 2. The kinetics of structural change $15 \%$ - tion solutions PABI. M - microscopy, $H_{v}$ - light scattering with crossed, $V_{v}$. when parallel polaroids

The shear deformation of the solution leads to the transformation of the circular pattern of the scattering and the emergence of an elliptical diffraction patterns corresponding to the predominant orientation of cylindrical domains deformation direction (Fig. 2, b). Precipitation-based solution in water is accompanied by a decrease in the size of the ellipse and the formation of the equatorial scattering reflexes at high angles, which are located along the direction of the initial deformation of the solution appearance of such diffraction patterns can be explained by analogy with those observed for the PPTA. Increased shear strain $\lambda_{\mathrm{s}}$ half is split LC phase domains into smaller anisometric structure is perpendicular to the direction of shear. Subsequent precipitation of water does not lead to a qualitative change in the diffraction patterns, in addition to a displacement of reflexes in the region of small scattering angles, which corresponds to an increase in the size of the anisotropic structures. Analysis of diffraction patterns shows that all the basic laws of structure found in the deposition of anisotropic PPTA solution, hold for anisotropic solutions $\mathrm{PABI}$ in $\mathrm{H}_{2} \mathrm{SO}_{4}$.
It is of interest to determine the boundary of the LC status in PABI system $-\mathrm{H}_{2} \mathrm{SO}_{4}$ on polymer concentration and temperature, as well as study the kinetics of the system's transition to the isotropic state when heated and the emergence of an anisotropic phase when cooled. Studies were carried out on solutions with a concentration of $10-20 \%$. In this case we use the full range of experimental techniques allowing the study of the temperature dependence of the scattering and extinction of paintings, as well as the overall intensity of the transmitted and scattered sample of polarized light. To select the optimal temperature conditions into account recommendations from the study of phase transitions in anisotropic solutions the PPTA. Phase transitions of the samples of anisotropic PABI (A) in the isotropic (I) state and vice versa, occur with certain combinations of the values of the critical concentrations and temperatures. It is possible to determine the status of the LC on the phase diagram of the system (Fig. 3).

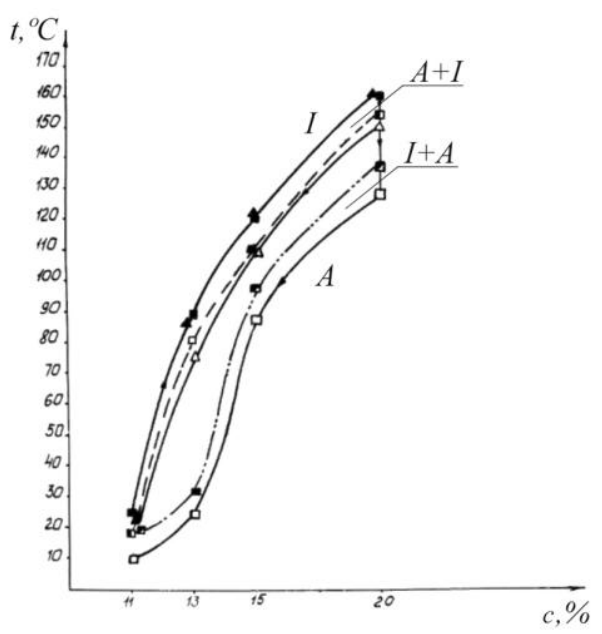

Fig. 3. Planning phase diagram $\mathrm{PABI}-\mathrm{H}_{2} \mathrm{SO}_{4}$ : - - the transition from the $\mathrm{LC}$ to the isotropic state (I) by heating, registered microscopy, $\square-$ on the diffraction of light; $\boldsymbol{\Lambda}$ appearance anisotropic phase (A) under cooling registrant microscopy, $\Delta$ - diffraction light.

Used for plotting points disappearance anisotropic phase when heated, and its appearance upon cooling by microscopy and recorded by light diffraction. The sample temperature is changed near the transition region at a rate of $0,5 \mathrm{dg} / \mathrm{min}$. As seen in Fig. 3, liquid crystal $\leftrightarrow$ isotropic liquid (LC $\leftrightarrow$ IL) phase transitions during heating and cooling are carried out for each of the combined concentrations in a certain temperature range The intensity of the transmitted polarized light to $11 \%$ solutions decreases monotonically with increasing temperature (Figure 4, curve 1). For the $13 \%$ solution is observed the appearance of a maximum intensity at a temperature of $63{ }^{\circ} \mathrm{C}$ (Fig. 4, curve 2), and for 15 and $20 \%$ - tion solutions - two peaks (Fig. 4, curve 3,4). The appearance of a maximum at lower temperatures can be attributed to the melting of crystal solvates. However, it must be borne in mind that extreme dependence of transmittance intensity can be caused by heating the mesophase transitions LC solutions. 


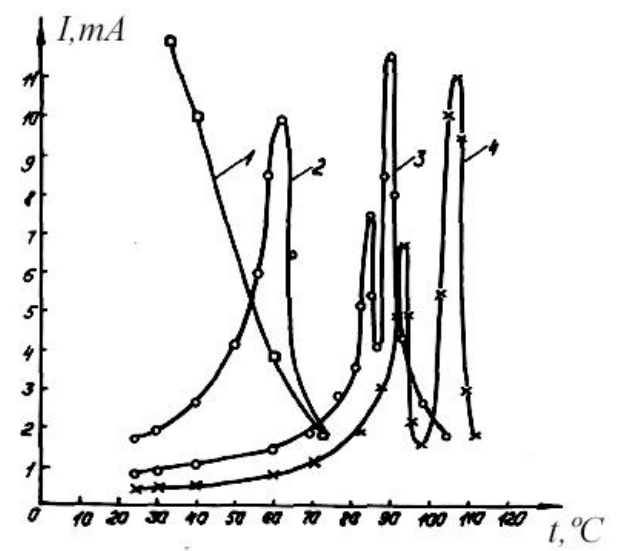

Fig. 4. Temperature the intensity of polarized light passing PABI sulfuric acid solutions of different concentrations, $\% 11$ (1) 13 (2) 15 (3), 20 (4).

To answer this question let us analyze the kinetics of structural changes in the phase transition LC $\leftrightarrow$ IL (Fig. 2). Heating LC sulfuric acid solutions PABI accompanied by a change in the color of the drug crossed nicols microscope (Fig. 2, M). Bright color is preserved up to $80{ }^{\circ} \mathrm{C}$ (a) $15 \%$-s' anisotropic solutions. Separation occurs at $80{ }^{\circ} \mathrm{C}$ on a yellow color, red, green strip (Fig. 2, M, b). Stained bands varies with rotation of the microscope stage, which indicates the absence of optical activity and typical of the nematic liquid crystals. An increase in temperature leads to a red color $\left(100{ }^{\circ} \mathrm{C}\right)$ of the drug $(\mathrm{M}, \mathrm{c})$, which disappears completely (118 ${ }^{\circ} \mathrm{C}$ ) at the transition to the isotropic state (Fig. 2, M, d). Upon cooling, the drug is an alternation of colors in reverse order, but at slightly lower temperatures. Such changes, but other temperatures observed during heating and cooling of the solutions in the other concentrations $(11,13,16,20 \%)$.

It is known that light scattering method in conjunction with other techniques allows to classify the type of liquid crystal and to study changes in the structure [4]. By virtue of the specific interaction with the molecule of $\mathrm{H}_{2} \mathrm{SO}_{4} \mathrm{PABI}$ arranged in a certain order to form domains that are commensurate with the length of the incident light. Reduction of the scattering angle $\theta$ indicates an increase in domain size and appearance of the scattering intensity depending on the azimuthal angle $\mu$ may be associated with a transition to a different texture LC.

Upon heating the solution several angle $\theta$ increases, the size of which corresponds to a reduction of ordered regions of liquid crystal. Existing in a certain temperature range anisometric structure (ribbon-shaped inclusions of different colors visible, and light scattering (Fig. 2, M, b). At these temperatures, the diffraction patterns become elliptical form (Fig. $H_{v}, V_{v}, \mathrm{~b}$ ). Changes in the nature of the orientation of the structural units from a disordered to an ordered, manifested in the transition from circular to elliptical scattering pattern. This may be due, in analogy with low molecular weight liquid crystal [5], the existence of a temperature gradient between the cell substrates with anisotropic PABI solution, which leads to the preferential orientation of the molecules. Areas of anisotropic and isotropic phases coexistence correspond significant changes in the intensity and shape of the scattering of paintings (Fig. 2, $H_{v}, V_{v}$, b). Near the transition to the isotropic phase the intensity of the diffraction pattern becomes dependent on the azimuth angle $\mu$ (Fig. $2, H_{v}, V_{v}$, c). This intensity distribution can be called molecular groups in a cholesteric state [3].

Heating and cooling rate not only change the temperature range of existence of phases, but also affects the structure of the sample. For example, the rapid cooling rate of $10{ }^{\circ} \mathrm{C} / \mathrm{min}, 15 \%$ solution, heated to $20-$ $30{ }^{\circ} \mathrm{C}$ above the transition temperature to the isotropic state, leads to the formation of the anisotropic phase in the form of diamond-shaped inclusions.

Loss LC embryos observed in a similar form of low molecular weight smectic liquid crystals [6, p.11]. However, based on this phenomenon can not assert a priori about the transition to the smectic mesophase, since when heated to these temperatures degradation of the polymer is also possible, which may lead to the formation of structures of this type on crystallization of degradation products. On the validity of the latter indicates a sharp decrease in the intrinsic viscosity of the system. It was also found a difference in the structure of anisotropic solutions of different concentrations PABI. $20 \%$ solutions formed in a temperature range of symmetric $H_{v}$ scattering pattern with peaks at an angle $\mu$ $=45^{\circ}$ to the direction of polarization. Diffraction patterns with azimuthal dependence of a different type ( $\mu$ $=0$ and $90^{\circ}$ ) were obtained upon cooling $15 \%$ solutions. As noted, a similar distribution of scattered light intensity in the direction corresponding to scattering of light from bar-like structures with main polarizability axis oriented at an angle of $45^{\circ}$ to the axis of the rod.

Observed by optical methods polymorphic transformations are also recorded on DTA thermograms (Fig. 5).

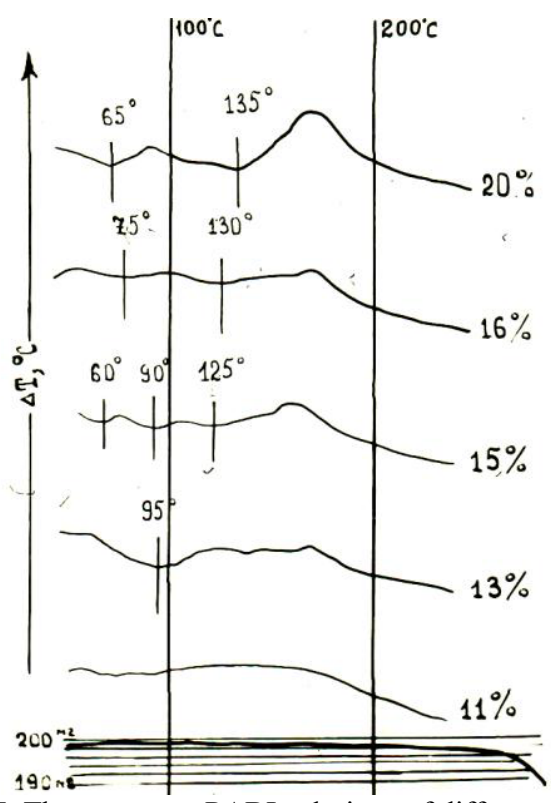

Fig. 5. Thermograms $\mathrm{PABI}$ solutions of different concentrations. The heating rate $2^{\circ} \mathrm{C} / \mathrm{min}$. 
The cycle of heating (cooling) LC PABI solutions of different concentrations are observed in the thermograms small in size (1 cal/gram) endoeffects showing the restructuring of the liquid crystal by heating. Maximum peak (5 cal $/ \mathrm{g}$ ) endothermic region with decreasing concentration of the solution is displaced by $140{ }^{\circ} \mathrm{C}$ (20\% solution) to $95{ }^{\circ} \mathrm{C}(13 \%$ strength $)$ and not accompanied by changes in sample weights. This shift corresponds to the transition LC $\leftrightarrow$ IL by heating the solution.

\section{Conclusions}

By using polarization-optical methods and the structural features of biphasic and anisotropic areas and built plots the phase diagram of the concentrated solutions PABI in $\mathrm{H}_{2} \mathrm{SO}_{4}$. It is found that to obtain an LC-based systems can be directly PABI solution PABI $\mathrm{H}_{2} \mathrm{SO}_{4}$ at a concentration above $11 \%$.Thermogram, as well as optical findings indicate that structural changes LC PABI solutions occur in a temperature range which increases with increasing concentration of polymer in solution. Under the influence of the temperature in the system may be structural changes associated with melting of the crystal solvates, as well as transitions from one to another modification of the LC, similar to that observed in [7]. Use of $\mathrm{H}_{2} \mathrm{SO}_{4}$ as a solvent alters not only the temperature - concentration phase transitions, but also the structure of anisotropic solutions PABI. In the transition PABI solutions in the anisotropic state during cooling are formed spherulites. Wherein in certain temperature - concentration of cooling modes can be observed the coexistence of three phases: isotropic crystal and a liquid crystal, which is shown as a characteristic of liquid crystal birefringent domains. As already mentioned, there are crystal solvates [8] at high concentrations of polymer solutions in sulfuric acid [8]. The regularities enable the correct selection of the conditions of implementation of the mesophase in other practically important polymers.

\section{References}

1. K.E. Perepelkin, The reinforcing fibers and fibrous polymer composites (Moscow, Publishing House of Scientific basis and technology, 2009)

2. SP. Papkov, VG Kulichikhin, The liquid crystal polymer state (Moscow, Chemistry, 1977)

3. O.A. Khanchich, S.A. Kuznetsova, Cloud of Science, 1, 30-32 (2013)

4. O.A. Khanchich, Anisotropic structure in polymers with varying rigidity of macromolecules (Thesis of Doct. Chem. Sciences, Moscow, 1997).

5. P.G. De Gennes, The Physics of Liquid Crystals (Mosocw, Mir, 1977)

6. I.G. Chistyakov, Liquid crystals (Moscow, Nauka, 1966)

7. E.M. Antipov, S.D. Artamonov, I.A. Volegova, J.K. Godowsky, Vysokomolek. Comm. A, 37(5), 800-810 (1995)

8. V.G. Kulichikhin, N.V. Vasileva, V.A. Platonov et al., Vysokomol. Comm. A, 18(3), 590-596 (1976) 